\title{
DEVELOPMENT OF GENERALIZED BLOCK CORRECTION PROCEDURES FOR THE SOLUTION OF DISCRETIZED NAVIER-STOKES EQUATIONS
}

\author{
Kanchan M. Kelkar and Suhas V. Patankar \\ University of Minnesota \\ Minneapolis, Minnesota
}

Computational methods are being increasingly used for the prediction of complex multidimensional flow phenomena. Although computations are far less expensive than full scale testing, costs of computational runs are still substantial. Therefore, considerable research effort is directed towards improving the accuracy and the efficiency of computational methods so that accurate predictions can be made at reasonable costs.

Computational methods involve discretization of the governing differential equations on a grid over the domain of interest. This generates a set of coupled nonlinear algebraic equations. Iterative methods are frequently used to solve these equations. However, iterative methods are prone to slow convergence or divergence and tend to be very sensitive to underrelaxation factors. Direct methods described in references [1], [2] and [3] do not suffer from these drawbacks. Accurate predictions of flow phenomena require use of fine grids. Direct methods cannot be used for such fine grid computations because of the excessive memory and cpu time requirements. In the present study, effort is directed towards developing a solution method which combines advantages of both the iterative and the direct methods. It involves iterative solution on the fine grid, convergence of which is enhanced by a direct solution for correction quantities on a coarse grid. 


\section{Nomenclature}

$a_{i, j}^{p}, a_{i, j}^{n b}, A$ coefficients in the fine grid discretization equation at grid point $(i, j)$

$\overline{\mathrm{a}}_{\mathrm{I}, \mathrm{J}}^{\mathrm{p}}, \overline{\mathrm{a}}_{\mathrm{I}, \mathrm{J}}^{\mathrm{nb}}, \mathrm{A}$ coefficients in the block correction equation for block (I,J)

$b_{i, j}$ source term in the fine grid discretization equation at grid point $(i, j)$

$b_{i, j}$

$\mathrm{p}_{\mathrm{i}, \mathrm{j}}$ source term in the block correction equation for block $(I, J)$ pressure at grid point $(i, j)$

$\overline{\mathrm{p}}_{\mathrm{I}, \mathrm{J}}$ uniform correction for pressure correction block $(I, J)$

$\mathrm{u}_{\mathrm{i}, \mathrm{j}}$ $\mathrm{u}$-velocity at grid point $(\mathrm{i}, \mathrm{j})$

$\overline{\mathrm{u}}_{\mathbf{l}, \mathrm{J}}$ uniform correction for u-velocity correction block (I,J)

\section{Formulation of the Block Correction Procedure}

The central feature in solving the discretization equations for flow prediction is the pressure-velocity coupling between the momentum and the continuity equations. Iterative methods solve the momentum and continuity equations in a sequential or an uncoupled manner. In addition, for iterative methods, the rate of propagation of the boundary information into the interior slows down as the grid fineness increases. Therefore, convergence of iterative methods is slow and it deteriorates as the grid fineness increases. In direct methods, all the equations are solved simultaneously so that the coupling between the continuity and the momentum equations is treated very effectively. The propagation of the boundary information into the interior is also instantaneous and iterations are performed only to account for the nonlinearity. Therefore, convergence for direct methods is fast and independent of the grid size. However, direct methods cannot be used for fine grids because of the excessive memory and cpu time requirements. The block correction technique described here has the low storage requirements of the iterative methods and the robustness of the direct methods. It uses iterative method to solve the equations on the grid on which the solution is sought, henceforth termed as fine grid. When the convergence of the iterative solution on the fine grid slows down, corrections to the existing solution are sought. The corrections are based on a coarse grid. The equations for correction quantities are solved directly so that the 
correction quantities converge rapidly. In the following subsections, derivation of the block correction equations and the details of the solution algorithm are discussed.

\section{Derivation of the block correction equations}

Discretization equations on the fine grid are constructed using the power law scheme described in reference [4]. A staggered mesh arrangement is used to store the velocity components. Coarse grid corrections to the existing values of fine grid variables are sought when the convergence of the iterative solution on the fine slows down.

The coarse correction grid is composed of correction blocks. Correction blocks for each variable are obtained by grouping the fine grid control volumes for that variable. Thus, $\mathrm{u}$-velocity correction blocks are obtained by grouping the $\mathrm{u}-$ momentum control volumes on the fine grid. Figure 1 illustrates the correction blocks for u-velocity, v-velocity and pressure. Since, $u$ and v-momentum control volumes are staggered with respect to the control volumes for pressure(continuity), correction blocks for these variables also exhibit similar arrangement. For each variable, a uniform correction is assumed over each correction block and this uniform correction is added to the values of all the fine grid unknowns of that variable whose control volumes belong to the correction block under consideration. Then, for each variable, the equations for the block corrections are obtained by simply summing the corrected fine grid discretization equations over each of the individual correction blocks. For example, a typical discretized u-momentum equation on the fine grid for velocity $u_{i, j}$ is

$$
a_{i, j}^{p} u_{i, j}=\sum a_{i, j}^{n b} u_{i, j}^{n b}+A\left(p_{i-1, j}-p_{i, j}\right)+b_{i, j}
$$

where $u_{i, j}^{n b}$ are the neighbor velocities of $u_{i, j}$. Now consider a $u$-velocity correction block $(\mathrm{I}, \mathrm{J})$, uniform correction on which is denoted by $\overline{\mathrm{u}}_{\mathrm{I}, \mathrm{J}}$. The corrections on the adjacent staggered correction blocks for pressure are denoted by $\overline{\mathrm{P}}_{\mathrm{I}-1, \mathrm{~J}}$ and $\overline{\mathrm{p}}_{\mathrm{I}, \mathrm{J}}$. The equation for this correction velocity is obtained by adding all the fine grid umomentum equations which belong to this correction block and has the following form. 


$$
\overline{\mathrm{a}}_{\mathrm{I}, \mathrm{J}}^{\mathrm{p}} \overline{\mathrm{u}}_{\mathrm{I}, \mathrm{J}}=\sum \overline{\mathrm{a}}_{\mathrm{I}, \mathrm{J}}^{\mathrm{nb}} \overline{\mathrm{u}}_{\mathrm{I}, \mathrm{J}}^{\mathrm{nb}}+\overline{\mathrm{A}}\left(\overline{\mathrm{p}}_{\mathrm{I}-1, \mathrm{~J}}-\overline{\mathrm{p}}_{\mathrm{I}, \mathrm{J}}\right)+\mathrm{b}_{\mathrm{i}, \mathrm{j}}
$$

The coefficients in the correction equation represent appropriate sums of the fine grid coefficients. In particular, the source term $b_{i, j}$ is given by

$$
b_{i, j}=\sum_{\substack{\text { correction } \\ \text { block }}}\left[\sum_{a_{i, j}}^{n b} u_{i, j}^{n b}+A\left(p_{i-1, j}-p_{i, j}\right)+b_{i, j}-a_{i, j}^{p} u_{i, j}\right]
$$

Thus, the source term is exactly equal to the algebraic sum of the residuals of all the u-momentum equations which belong to the correction block for u-velocity. Similar equations can be written for v-velocity block corrections. Equations for correction pressures are obtained by summing the corrected fine grid continuity equations over the correction blocks for pressure.

Equations for the velocity and pressure block corrections exhibit two important characteristics. Firstly, the form of the correction equations is exactly the same as that of the discretization equations on the fine grid. Secondly, all the correction equations are driven by the residuals on the fine grid. Thus, when the fine grid solution is converged, the corrections to the fine grid field become zero automatically.

\section{The solution algorithm}

The overall solution algorithm employed is very similar to the multigrid solution procedure of reference 5 with two grid levels and is shown in figure 2. Starting with an initial guess, coefficients for the fine grid discretization equations are computed. With these values of the fine grid coefficients, coeffients for the block correction equations are computed. The Yale Sparse Matrix Package, which uses LU factorization, is employed to solve the block correction equations directly and the values of the fine grid variables are corrected appropriately. To account for the nonlinearity, this procedure is repeated till the predicted corrections are small. Since, a direct solution method is employed to solve the correction equations, these iterations converge very rapidly. The corrected fine grid field satisfies integral balances of momentum and mass over the correction blocks. The 
high frequency errors which now remain in the fine grid field are removed by solving the fine grid equations using the SIMPLER algorithm described in reference 4 . When the rate of residue reduction per iteration on the fine grid drops below 0.5 , coarse grid corrections are sought. This switching between the fine grid solution and coarse grid corrections is repeated till convergence.

\section{Results and Discussion}

The proposed block correction procedure has been applied to compute recirculating flow in a driven cavity. The flow in a driven cavity is strongly elliptic with significant nonlinearities at $\mathrm{Re}=2000$ and it provides a good test for evaluating the performance of the block correction procedure. Figure 3 shows the variation of the fine grid residual with the number of iterations performed for a fine grid size of $62 \times 62$. Note that the iterations represent the repetitions of the SIMPLER algorithm on the fine grid irrespective of whether the block correction procedure is employed or not. It can be seen that the rate of residual reduction for SIMPLER alone is low and it decreases as the computation proceeds. However, when the block correction procedure is employed in conjunction with SIMPLER on the fine grid, the rate of residual reduction is substantially higher and is maintained at that high value till convergence. As the fineness of the block correction grid increases, the residue reduces at a faster rate, but with an associated increase the effort required for computing the corrections. Thus, for a given size of the fine grid, there is an optimum size of the correction grid for which the computational effort required to obtain the converged solution is minimized. Table 1 lists the number of fine grid iterations and the corresponding computational effort for two different fine grid sizes for SIMPLER with and without block corrections. Note that as the the main grid becomes finer, the number of iterations required for the SIMPLER algorithm increase substantially. But, when the block correction procedure is utilized, the number of fine grid iterations remain almost constant. Thus, although for a grid size of $20 \times 20$, the use of block correction procedure does not reduce the computational effort over that required for SIMPLER alone, substantial savings are obtained for the fine grid size of $62 \times 62$.

The proposed block correction procedure can also be effectively utilized to facilitate the convergence of subdomain methods. Subdomaining is often employed to minimize wasteful computations when the geometries for which the flow 
computations are being made, include large passive areas. Boundary conditions on the internal boundaries of the subdomains are not known a priori and have to be adjusted iteratively. Convergence of the subdomain methods is dependent critically on the correctness of these boundary conditions. Coarse grid corrections spanning the entire domain can provide a good guess for the global solution and hence a good guess for the subdomain boundary conditions. Thus, the use of the block correction procedure can enhance the convergence of subdomain methods as well as remove the need for substantial overlapping among subdomains that is otherwise present. Work is currently in progress to verify the validity of this approach.

\section{References}

1. Braaten M. E. : Development and Evaluation of Iterative and Direct Methods for the Solution of the Equations Governing Recirculating Flows. Ph.D Thesis, University of Minnesota, 1985.

2. Vanka S. P., Leaf G. K. : Fully-Coupled Solution of Pressure-Linked Fluid Flow Equations. Argonne National Laboratory Report: ANL-83-73, 1983.

3. J. Ward MacArthur : Development and Implementation of Robust Direct Finite Difference Methods for the Solution of Strongly Coupled Elliptic Transport Equations. PhD Thesis, University of Minnesota, 1986.

4. Patankar S. V. : Numerical Heat Transfer and Fluid Flow. Hemisphere Publishing Corporation, 1980.

5. Brandt A. : Multi Level Adaptive Solutions to the Boundary Value Problems. Math. Comp., Vol. 31, 1980. 
Table 1 - Number of iterations and the computational effort required for the solution of the driven cavity problem for $\mathrm{Re}=2000$ with and without block correction

\section{Iterations(Computer Time*)}

Driven Cavity $(\mathrm{Re}=2000)$

\begin{tabular}{cccc} 
Main Grid & SIMPLER & $\begin{array}{c}\text { Correction } \\
\text { grid }\end{array}$ & $\begin{array}{c}\text { SIMPLER with } \\
\text { block correction }\end{array}$ \\
\hline $20 \times 20$ & $42(1.1)$ & $7 \times 7$ & $22(3.0)$ \\
\hline $62 \times 62$ & $250(51.0)$ & $21 \times 21$ & $27(21.0)$ \\
\hline
\end{tabular}

* CPU seconds on CRAY-1 supercomputer 
$\square$ correction block for pressure

$\square$ correction block for $u$ velocity

i correction block for $v$ velocity

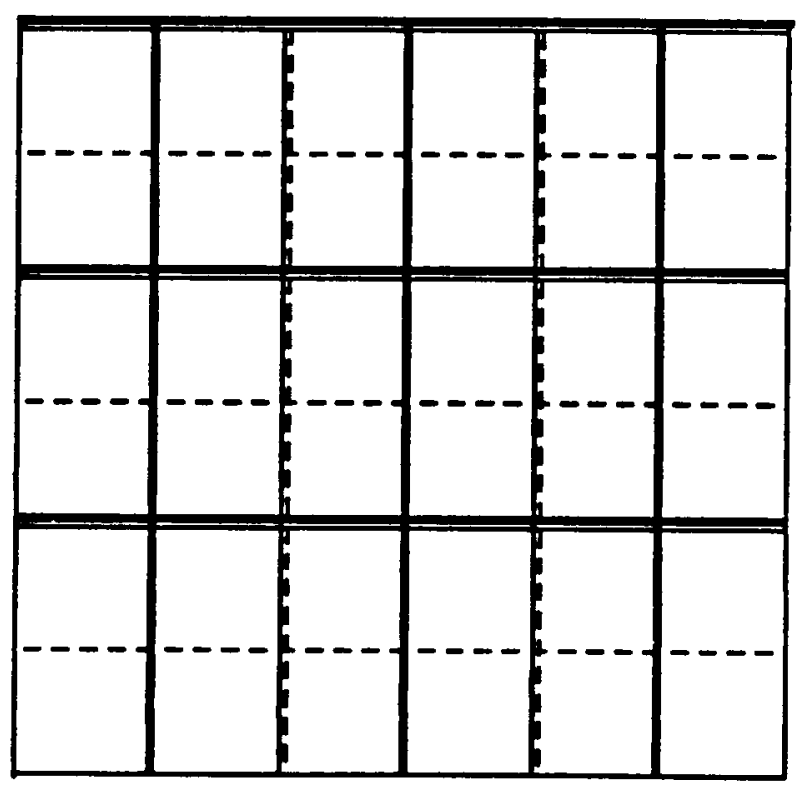

Figure 1 - Staggered arrangement of the correction blocks 


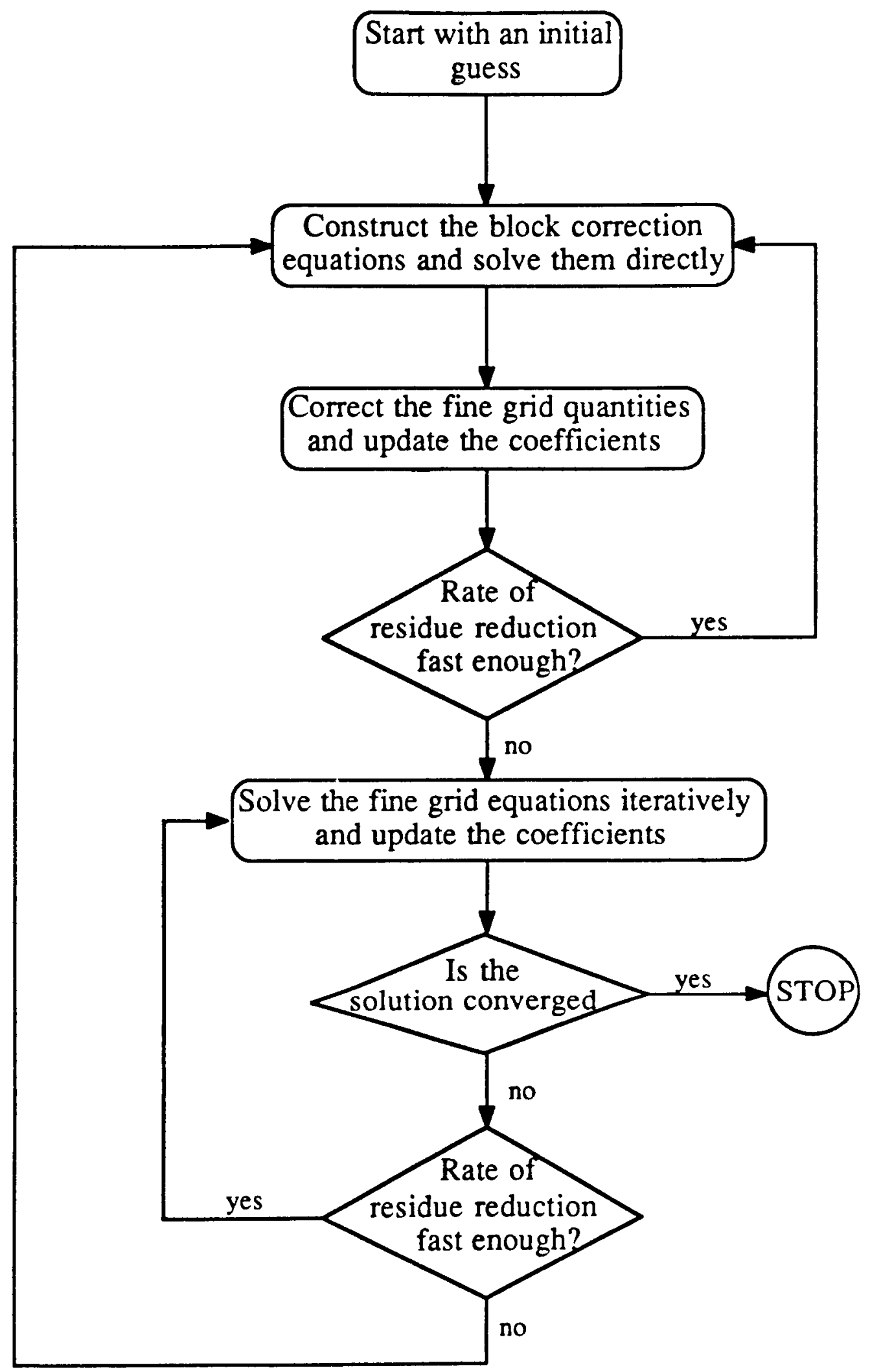

Figure 2 - The solution algorithm 


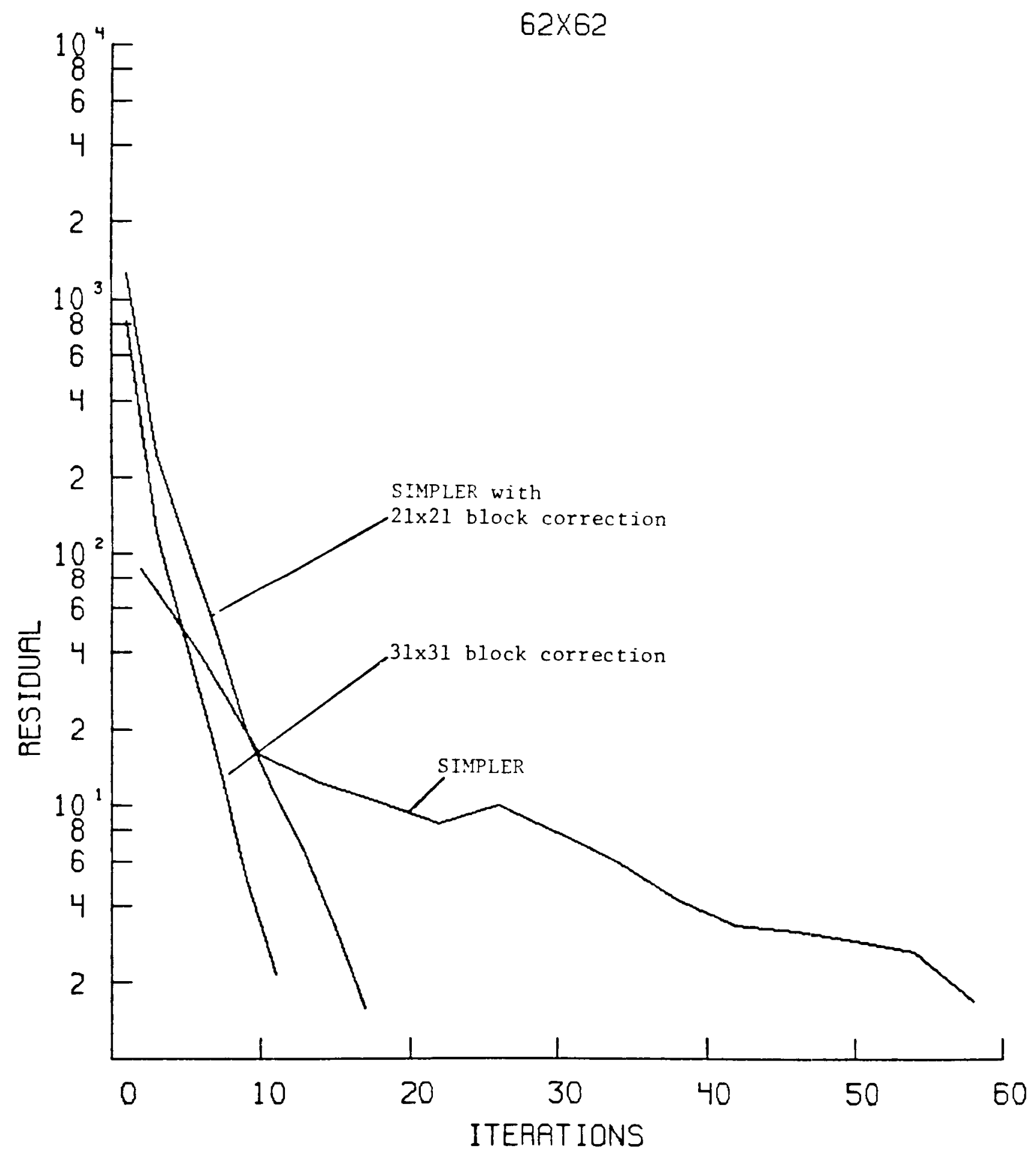

Figure 3 - Variation of the fine grid residual with the number of iterations on the fine grid for the driven cavity problem with $\mathrm{Re}=2000$ 